EPJ Web of Conferences 66, 04010 (2014)

DOI: $10.1051 /$ epjconf/ 20146604010

(C) Owned by the authors, published by EDP Sciences, 2014

\title{
From microscopic interactions to the dynamics of the fireball
}

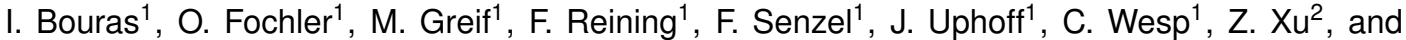 \\ C. Greiner ${ }^{1 a}$ \\ ${ }^{1}$ Institut für Theoretische Physik, Johann Wolfgang Goethe-Universität, Max-von-Laue-Str. 1, D-60438 \\ Frankfurt am Main, Germany \\ ${ }^{2}$ Department of Physics, Tsinghua University, Beijing 100084, China
}

\begin{abstract}
We discuss recent applications of the partonic perturbative QCD based cascade model BAMPS with focus on heavy-ion phenomenology in the hard and soft momentum range. First, the elliptic flow and suppression of charm and bottom quarks are studied at LHC energies. Thereafter, we compare in a detailed study the standard Gunion-Bertsch approximation of the matrix elements for inelastic processes to the exact results in leading order perturbative QCD. Since a disagreement is found, we propose an improved GunionBertsch matrix element, which agrees with the exact result in all phase space regions.
\end{abstract}

\section{Introduction}

Recent experimental and theoretical results from heavy-ion collisions at ultrarelativistic energies carried out at the Relativistic Heavy-Ion Collider (RHIC) and the Large Hadron Collider (LHC) indicate that a new state of matter, the quark-gluon plasma (QGP), with a very short life time is created.

The large values of the measured hadronic elliptic flow $v_{2}$ [1] suggests that equilibration of quarks and gluons occurs on a very short time scale $\leq 1 \mathrm{fm} / \mathrm{c}$. This also suggests that the shear viscosity over entropy density ratio $\eta / s$ of the QGP is very small, which means that the QGP behaves like a nearly perfect fluid. All these conclusions can be drawn from comparison of experimental results with hydrodynamic calculations. However, an understanding of the mechanism of fast thermalization cannot be achieved in the scope of hydrodynamic models. The early pre-equilibrium dynamics of the QGP must be studied on the microscopic level.

In contrast to the hydrodynamic approach, kinetic transport theory is a microscopic theory and thus allows to study processes of soft and hard processes simultaneously. This is in particular important for detailed understanding of further properties of the quark-gluonic medium, such as the suppression of jets and heavy-quarks. Suppression of jets, also known as jet quenching, is quantified by comparing the hadron multiplicities measured in heavy-ion collisions with appropriately scaled multiplicities from $p+p$ collisions [2]. In addition, very exciting jet-associated particle correlations were observed [3], which might be the result of a conical emission off propagating shock waves in form of Mach Cones. These Mach Cones might be induced by high-energy partons traversing the expanding medium [4]. However, recent studies show that the most promising explanation for the appearing double-peak

\footnotetext{
ae-mail: carsten.greiner@th.physik.uni-frankfurt.de
} 
structure are fluctuating initial conditions [5]. Nevertheless, the issue about Mach cones is yet not clarified and demands a deeper investigation.

The partonic transport model BAMPS (Boltzmann Approach to MultiParton Scatterings) [6] has been developed to provide a unified description of the dynamics of the early QGP stage of heavy-ion collisions (HIC) including perturbative QCD based elastic and inelastic processes. BAMPS has been applied to provide explanations of fast thermalization on a very short time scale $\leq 1 \mathrm{fm} / \mathrm{c}$ [7] as well as a small value of $\eta / s \approx 0.08-0.2$ for $\alpha_{s}=0.6-0.3[8,9]$, calculated with the original GunionBertsch (GB) matrix element for inelastic processes. However, with extensive numerical comparisons between the GB approximation and the exact leading order pQCD result we showed that the original Gunion-Bertsch (GB) deviates from the exact result at forward and backward rapidity [10]. To this end, we proposed an improved version of the GB matrix element that cures the problems within the GB approximations and, thus, is valid in all regions of phase space. BAMPS calculations with the original GB matrix element provide results on elliptic flow [11,12] and jet quenching [13] at RHIC energies. New calculations for various observables with the improved GB matrix element are underway.

In these proceedings we present BAMPS results on elliptic flow and suppression of charm and bottom quarks for LHC energies in Sec. 2. In Sec. 3 we show the comparison of the Gunion-Bertsch approximation to the exact result within leading order perturbative QCD.

\section{Elliptic flow and suppression of heavy quarks}

Heavy quarks are a good probe to study the properties of the QGP. They are accurately calibrated due to their well known production process. Furthermore, heavy quarks are produced entirely in the early stage of the heavy ion collision due to their large mass [14] and are also tagged during hadronization due to flavor conservation.

All the calculations for heavy quarks in this section are done with a running coupling and an improved Debye screening. The latter means that the screening mass of the $t$ channel of elastic scatterings is determined such that the energy loss matches the energy loss of a heavy quark calculated within the hard thermal loop approach.

Detailed studies in BAMPS [15-17] show that elastic energy loss of heavy quarks alone is not compatible with the experimental data at RHIC and LHC. However, elastic energy loss explains a significant portion of the overall suppression. If we employ a running coupling and improved Debye screening the experimental data for both $v_{2}$ and $R_{A A}$ for both RHIC and LHC can be explained if the elastic cross section is multiplied with the artificial factor $K=3.5$. This indicates that radiative energy loss should be about two times larger than the elastic energy loss. However, this must be checked in a forthcoming study, which will be carried out with the improved Gunion-Bertsch matrix element from Sec. 3 generalized to heavy quarks. On the left hand side of Fig. 1 the nuclear modification factor of $D$ mesons for very central events at LHC is depicted. Our prediction with BAMPS is slightly smaller than the experimental data points. This is in agreement with the observation that we also underestimate the $R_{A A}$ of non-prompt $J / \psi$, heavy flavor electrons, and muons [17].

The right hand side of Fig. 1 shows our predictions of the elliptic flow of $D$ mesons, non-prompt $J / \psi$, heavy flavor electrons, and muons. For $D$ mesons and electrons there is already data available which agrees well with our calculations.

\section{Comparison of the Gunion-Bertsch cross section to the exact result}

A commonly used approximation to the leading order perturbative QCD matrix element for partonic $2 \leftrightarrow 3$ processes is a result derived by Gunion and BERTSCH (GB) [21]. When employing the matrix 

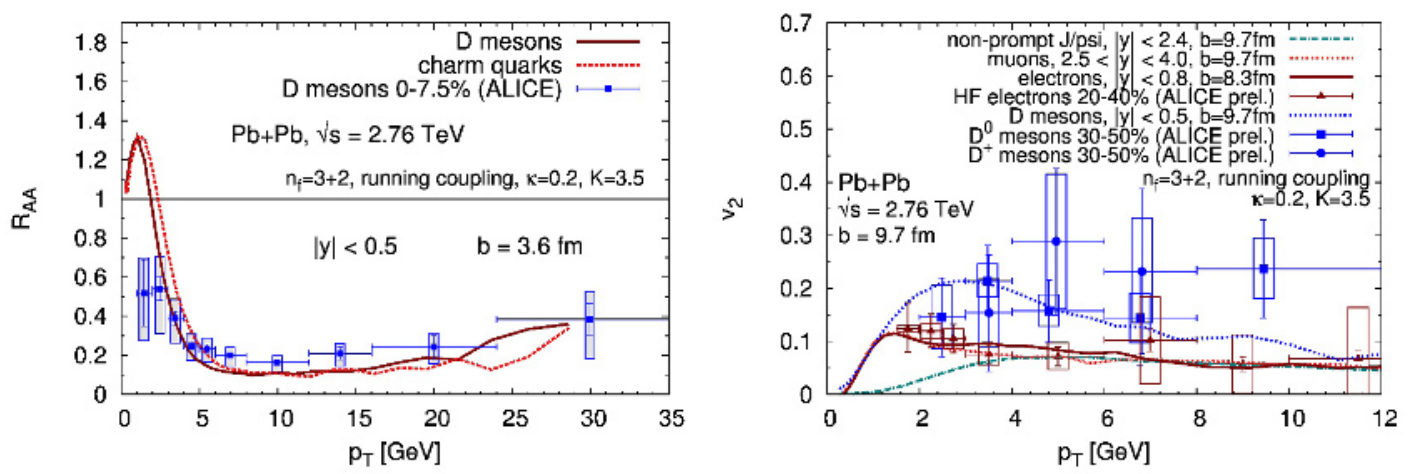

Figure 1. Left: Nuclear modification factor $R_{A A}$ of $D$ mesons for $b=3.6 \mathrm{fm}$ with data [18]. Right: Elliptic flow $v_{2}$ of $D$ mesons, non-prompt $J / \psi$, muons, and electrons at LHC together with available data for $D$ mesons and electrons $[19,20]$.

element to obtain rates or cross sections from phase space integration, the amplitude by GuNION and BERTSCH deviates from the exact result [22] in characteristic regions of the phase space [10], which leads to a sizeable deviation [23]. Therefore, we propose an improved version of the Gunion-Bertsch matrix element which agrees very well with the exact result in all phase space regions:

$$
\left|\overline{\mathcal{M}}_{q q^{\prime} \rightarrow q q^{\prime} g}\right|^{2} \simeq 12 g^{2}\left|\overline{\mathcal{M}}_{q q^{\prime} \rightarrow q q^{\prime}}\right|_{\mathrm{sa}}^{2}(1-\bar{x})^{2} \frac{q_{\perp}^{2}}{k_{\perp}^{2}\left(\mathbf{q}_{\perp}-\mathbf{k}_{\perp}\right)^{2}}
$$

where "sa" stands for small angle approximation, $g$ is the strong coupling, $k_{\perp}\left(q_{\perp}\right)$ the transverse momentum of the radiated (internal) gluon, and $\bar{x}=k_{\perp} \mathrm{e}^{|y|} / \sqrt{s}$ the symmetrized longitudinal momentum fraction of the emitted gluon. The derivation is explicitly shown in Ref. [10]. The difference to the original GB matrix element consists of two parts, a) keeping a kinematic factor $(1-x)^{2}$, where $x$ is the fraction of light cone momentum carried by the radiated gluon; and $b$ ) respecting the symmetry of the process by explicitly combining results from the two gauge choices $A^{+}=0$ and $A^{-}=0$, restricting the emission of gluons to the respective forward direction. The latter is necessary since the GB approximations are not applicable in the respective backward regions for both gauges [10]. In contrast to the original GB matrix element, the improved result is not only valid at mid-rapidity, but also at forward and backward rapidity.

The left panel of Fig. 2 compares the rapidity spectrum of the emitted gluon as given by different approximations of the matrix element by depicting the differential cross section $\mathrm{d} \sigma / \mathrm{d} y$ for the process $q q^{\prime} \rightarrow q q^{\prime} g$. Although the original GB matrix element agrees very well with the exact result at midrapidity (the region in which GuNION and Bertsch were mainly interested in) large deviations can be seen at forward and backward rapidity. Based on a detailed analytic investigation of the underlying approximations, our proposed improved version of the GB matrix element agrees very well in all phase space regions with the exact result. This remarkable agreement holds for virtually all center of mass energies $\sqrt{s}$ as well as for the other radiative processes $q g \rightarrow q g g$ and $g g \rightarrow g g g$, which is depicted on the right hand side of Fig. 2.

The implementation of the new improved GB matrix element in our transport model BAMPS is currently under way. As part of this study we will also implement the running coupling for elastic and radiative processes as well as radiative processes for heavy quarks in Sec. 2. 

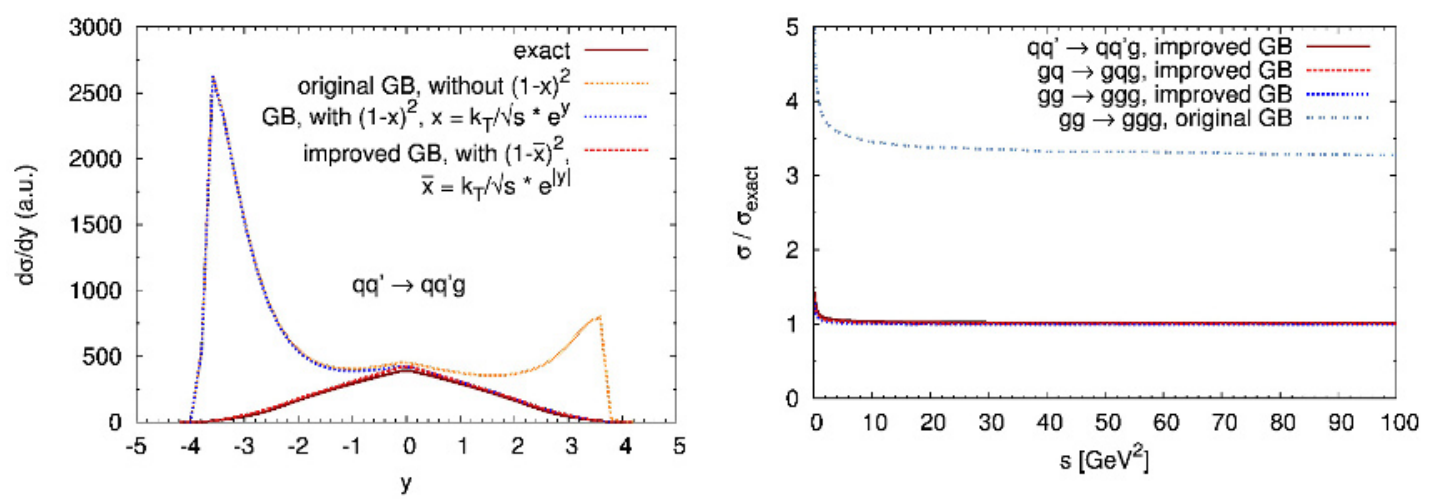

Figure 2. Left: Differential cross section $\mathrm{d} \sigma / \mathrm{d} y$ for the process $q q^{\prime} \rightarrow q q^{\prime} g$ calculated with the exact [22], original GB [21], GB with $(1-x)^{2}$, and improved GB with $(1-\bar{x})^{2}$ (Eq. (1)) matrix element. Right: Ratio of total cross section $\sigma_{2 \rightarrow 3}$ of the improved and original GB matrix elements to the exact one for different processes. For details see Ref. [10].

\section{References}

[1] S.S. Adler et al. (PHENIX), Phys. Rev. Lett. 91, 182301 (2003), nucl-ex/0305013

[2] J. Adams et al. (STAR), Phys. Rev. Lett. 91, 172302 (2003), nucl-ex/0305015

[3] F. Wang (STAR), J. Phys. G30, S1299 (2004), nucl-ex/0404010

[4] H. Stoecker, Nucl. Phys. A750, 121 (2005), nucl-th/0406018

[5] B. Schenke, S. Jeon, C. Gale, Phys.Rev.Lett. 106, 042301 (2011), 1009. 3244

[6] Z. Xu, C. Greiner, Phys. Rev. C71, 064901 (2005), hep-ph/0406278

[7] A. El, Z. Xu, C. Greiner, Nucl. Phys. A806, 287 (2008), 0712 . 3734

[8] Z. Xu, C. Greiner, Phys. Rev. Lett. 100, 172301 (2008), 0710. 5719

[9] A. El, A. Muronga, Z. Xu, C. Greiner, Phys. Rev. C79, 044914 (2009), 0812 . 2762

[10] O. Fochler, J. Uphoff, Z. Xu, C. Greiner (2013), 1302.5250

[11] Z. Xu, C. Greiner, H. Stöcker, Phys. Rev. Lett. 101, 082302 (2008), 0711.0961

[12] Z. Xu, C. Greiner, Phys. Rev. C79, 014904 (2009), 0811.2940

[13] O. Fochler, Z. Xu, C. Greiner, Phys. Rev. Lett. 102, 202301 (2009), 0806.1169

[14] J. Uphoff, O. Fochler, Z. Xu, C. Greiner, Phys. Rev. C82, 044906 (2010), 1003.4200

[15] J. Uphoff, O. Fochler, Z. Xu, C. Greiner, Phys.Rev. C84, 024908 (2011), 1104.2295

[16] A. Meistrenko, A. Peshier, J. Uphoff, C. Greiner, Nucl.Phys. A901, 51 (2013), 1204.2397

[17] J. Uphoff, O. Fochler, Z. Xu, C. Greiner, Phys.Lett. B717, 430 (2012), 1205.4945

[18] A. Grelli (ALICE Collaboration), Nucl.Phys.A904-905 2013, 635c (2013), 1210.7332

[19] G. Ortona (ALICE Collaboration) (2012), 1207.7239

[20] S. Sakai (ALICE collaboration) (2012), quark Matter 2012 proceedings

[21] J. Gunion, G. Bertsch, Phys.Rev. D25, 746 (1982)

[22] F.A. Berends, R. Kleiss, P. De Causmaecker, R. Gastmans, T.T. Wu, Phys. Lett. B103, 124 (1981)

[23] J.W. Chen, J. Deng, H. Dong, Q. Wang, Phys.Rev. C87, 024910 (2013), 1107.0522 